Letter to the Editor

\title{
Subretinal fluid and choroidal venous congestion secondary to a presumed dural cavernous sinus fistula
}

\author{
Dujon R.W. Fuzzard MBBS, ${ }^{1}$ Thomas J . Gin MBBS(Hons), ${ }^{1}$ Thomas G. Hardy \\ FRANZCO 2,3 and C. Alex Harper FRANZCO ${ }^{4,5}$
}

${ }^{1}$ Royal Victorian Eye and Ear Hospital, Melbourne, Victoria, Australia.

2Orbital, Plastic and Lacrimal Clinic, Royal Victorian Eye and Ear Hospital, Melbourne, Victoria, Australia.

${ }^{3}$ Department of Surgery, Royal Melbourne Hospital, The University of Melbourne, Melbourne, Victoria, Australia.

${ }^{4}$ Department of Ophthalmology, The University of Melbourne, Melbourne, Victoria, Australia.

5Medical Retina Clinic, Royal Victorian Eye and Ear Hospital, Melbourne, Victoria, Australia.

Correspondence: Dr Dujon Fuzzard, Royal Victorian Eye and Ear Hospital, 32 Gisborne Street, East Melbourne, Victoria, 3002, AUSTRALIA

Email: dujonfuzzard@gmail.com

Received 21 August 2019; accepted 28 J anuary 2020

Funding sources / Financial disclosure: None

Conflict of interest: None

This is the author manuscript accepted for publication and has undergone full peer review but has not been through the copyediting, typesetting, pagination and proofreading process, which may lead to differences between this version and the Version of Record. Please cite this article as doi: 10.1111/ceo.13732

This article is protected by copyright. All rights reserved. 


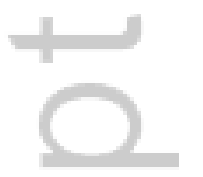


Manifestations of carotid-cavernous fistulae (CCF) that arise from orbital venous congestion are diverse. Enhanced-depth imaging optical coherence tomography (EDI-OCT) first linked increased choroidal thickness with radiologically-confirmed CCF in 2013. ${ }^{1}$ We describe multimodal imaging characteristics of a patient with increased choroidal thickness, serous retinal and retinal pigment epithelial (RPE) detachments as a complication of a presumed CCF.

A 62-year-old female presented with one month of central blur in her left eye, with a one-year history of bilateral conjunctival redness, epiphora and proptosis. Previous computed tomography with angiography (CTA) of the brain and orbits had shown bilateral proptosis, mild enlargement of the medial and inferior recti and symmetric superior ophthalmic veins, but no definitive evidence of CCF or other diagnosis. Visual acuity was slightly affected (right $6 / 6$; left $6 / 9$, improving to $6 / 6$ with +0.50 DS correction). Her intra-ocular pressure (IOP) was 18 and $20 \mathrm{mmHg}$ in the right and left eye respectively. There was bilateral proptosis ( $26 \mathrm{~mm}$ in each eye measured by Hertel exophthalmometry), prominent episcleral blood vessels and inferior scleral show (Figure 1A). Extraocular movements were full. Subretinal fluid was noted clinically at both maculae with no retinal haemorrhages or retinal vascular tortuosity.

Figure 1: External photography demonstrating prominent episcleral blood vessels in the right eye (1A). Fundus fluorescein angiography of the left eye, at 29 seconds, demonstrating marked delay of choroidal filling (1B). Indocyanine green angiography, at 44 (1C) and 32 seconds (1D), revealed bilateral patchy nonperfusion of the choriocapillaris and 'corkscrewing' of selective large choroidal vessels (1C\&D).

Fundus fluorescein angiography (FFA) demonstrated marked delay of choroidal filling with normal filling and transit of the retinal circulation (Figure 1B). There was 
pooling of fluorescein within a retinal pigment epithelial detachment (PED) in the right eye, but no leakage in the area of the serous detachment or from venous trunks. Indocyanine green angiography (ICGA) revealed bilateral patchy nonperfusion of the choriocapillaris and dilation and tortuosity ('corkscrewing') of selective large choroidal vessels (Figure 1C\&D). Macular optical coherence tomography (OCT) confirmed the presence of bilateral sub-retinal fluid and RPE clumping, with an associated PED in the right eye (Figure 2A\&B). Subfoveal choroidal thickness (SFCT) was defined and measured using the same criteria used by I nam et al. ${ }^{2}$ I nitial measurements (right $329 \mu \mathrm{m}$, left $372 \mu \mathrm{m}$ ) confirmed the presence of a thickened choroid.

Figure 2: High-resolution SD-EDI-OCT horizontal line scan through the fovea (Spectralis HRA+OCT, Heidelberg Engineering, Heidelberg, Germany) of the right (2A) and left (2B) eyes on presentation demonstrating bilateral sub-retinal fluid and a right pigment epithelial detachment. Enhanced depth imaging OCT of the right (2C) and left (2D) eyes, 7-months following initial presentation, showing resolution of subretinal fluid. SFCT measurements are displayed on each image.

Repeat CTA of the brain and orbits was suggestive of a dural cavernous sinus fistula. It demonstrated enlargement of the right superior ophthalmic vein with filling during the arterial phase and enlargement of extraocular muscles. Subsequent time-offlight magnetic resonance angiography (MRA) of the brain confirmed these indirect findings of a dural CCF, however neither imaging technique identified a clear arteriovenous shunt site. Cerebral digital subtraction angiography (DSA) was offered to the patient, which she declined due to the risk of stroke. She remained under observation. 
Seven months after presentation, uncorrected visual acuity had improved (right 6/4.8; left 6/6). EDI-OCT demonstrated resolution of subretinal fluid and the PED (Figure 2C\&D). SFCT (right $212 \mu \mathrm{m}$, left $273 \mu \mathrm{m}$ ) showed a corresponding reduction.

The diagnosis of CCF is frequently supported by conclusive neuro-radiological identification of the fistula tract, however such imaging is not without limitation. The relative sensitivity of CTA, MRA and cerebral DSA, to detect either the size or location of the fistula, has been reported to be $87 \%, 80 \%$ and $94 \%$ respectively. ${ }^{3}$ The features in this case of proptosis, episcleral venous dilatation, choroidal venous congestion, and premature filling of the right superior ophthalmic vein on CTA, mount a compelling case for the clinical diagnosis of a dural CCF. The subsequent resolution of subretinal fluid and PED in the right eye and corresponding reduction in SFCT is consistent with partial spontaneous closure of the fistula. Imam et al. ${ }^{2}$ recently demonstrated that EDI-OCT measurement of SFCT was elevated in angiographically proven CCF and may be a diagnostic aid. In clinically suspicious cases with inconclusive neuro-imaging, the diagnostic value of multimodal retinal and choroidal imaging may be of higher importance.

Serous macular detachments have been described as a rare manifestation of CCF. ${ }^{4}$ Consistent with the findings of Rey et $a l,{ }^{5}$ our patient exhibited increased subfoveal choroidal thickness in the presence of subretinal fluid on presentation, which reduced with resolution of subretinal fluid upon spontaneous partial closure of the fistula. Arterialization of orbital veins has been proposed to cause venous congestion, hypoxia of the choriocapillaris and subsequent impairment of RPE function. ${ }^{4}$ Our findings of delayed choroidal filling, delayed intrachoroidal circulation and patchy filling defects of the choriocapillaris support the presence of venous congestion, underpinning this hypothesis. 
This case documents imaging characteristics of bilateral choroidal venous congestion associated with a presumed dural cavernous sinus fistula. The abnormalities of structure and haemodynamics of the choroidal vasculature had spared the retinal circulation. The FFA and ICGA demonstrated marked delay in choroidal filling, delayed intrachoroidal circulation and patchy filling defects of the choriocapillaris. The arterialized orbital veins in both eyes showed selective dilatation and tortuosity, giving the choroidal vessels an appearance reminiscent of the 'corkscrew' episcleral vessels seen externally. In clinically suspicious cases with negative neuro-imaging studies, multimodal retinal and choroidal imaging may aid diagnosis.

\section{REFERENCES}

1. Shinohara Y, Kashima T, Akiyama H, Kishi S. Alteration of choroidal thickness in a case of carotid cavernous fistula: a case report and a review of the literature. BMC Ophthalmol. 2013;13:75.

2. Inam O, Arat YO, Yavas GF, Arat A. Retinal and Choroidal Optical Coherence Tomography Findings of Carotid Cavernous Fistula. Am J Ophthalmol. 2019;206:26473.

3. Chen CC-C, Chang PC-T, Shy C-G, Chen W-S, Hung H-C. CT Angiography and MR Angiography in the Evaluation of Carotid Cavernous Sinus Fistula Prior to Embolization: A Comparison of Techniques. AJ NR Am J Neuroradiol. 2005;26(9):2349-56.

4. Garg SJ, Regillo CD, Aggarwal S, Bilyk JR, Savino PJ. Macular exudative retinal detachment in a patient with a dural cavernous sinus fistula. Arch Ophthalmol. 2006; 124(8):1201-2. 
5. Rey A, Castillo L, Dyrda A, Maseras X, Jürgens I. Subfoveal choroidal thickness changes in carotid cavernous fistula following spontaneous resolution. BMC Ophthalmol. 2016;16:63.

This article is protected by copyright. All rights reserved. 


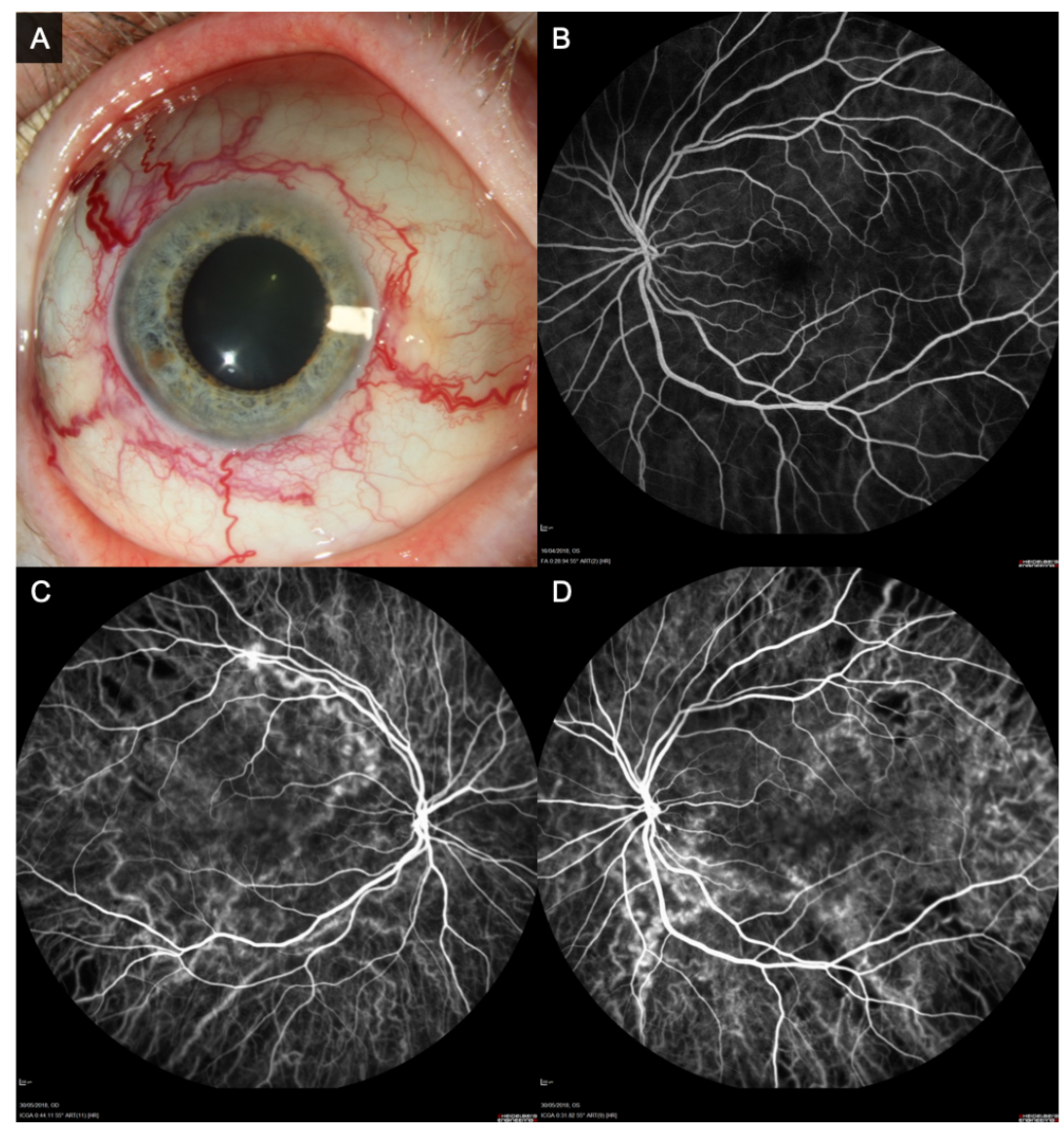

CEO_13732_CEO-19-08-0837 figure 1.tiff

This article is protected by copyright. All rights reserved. 


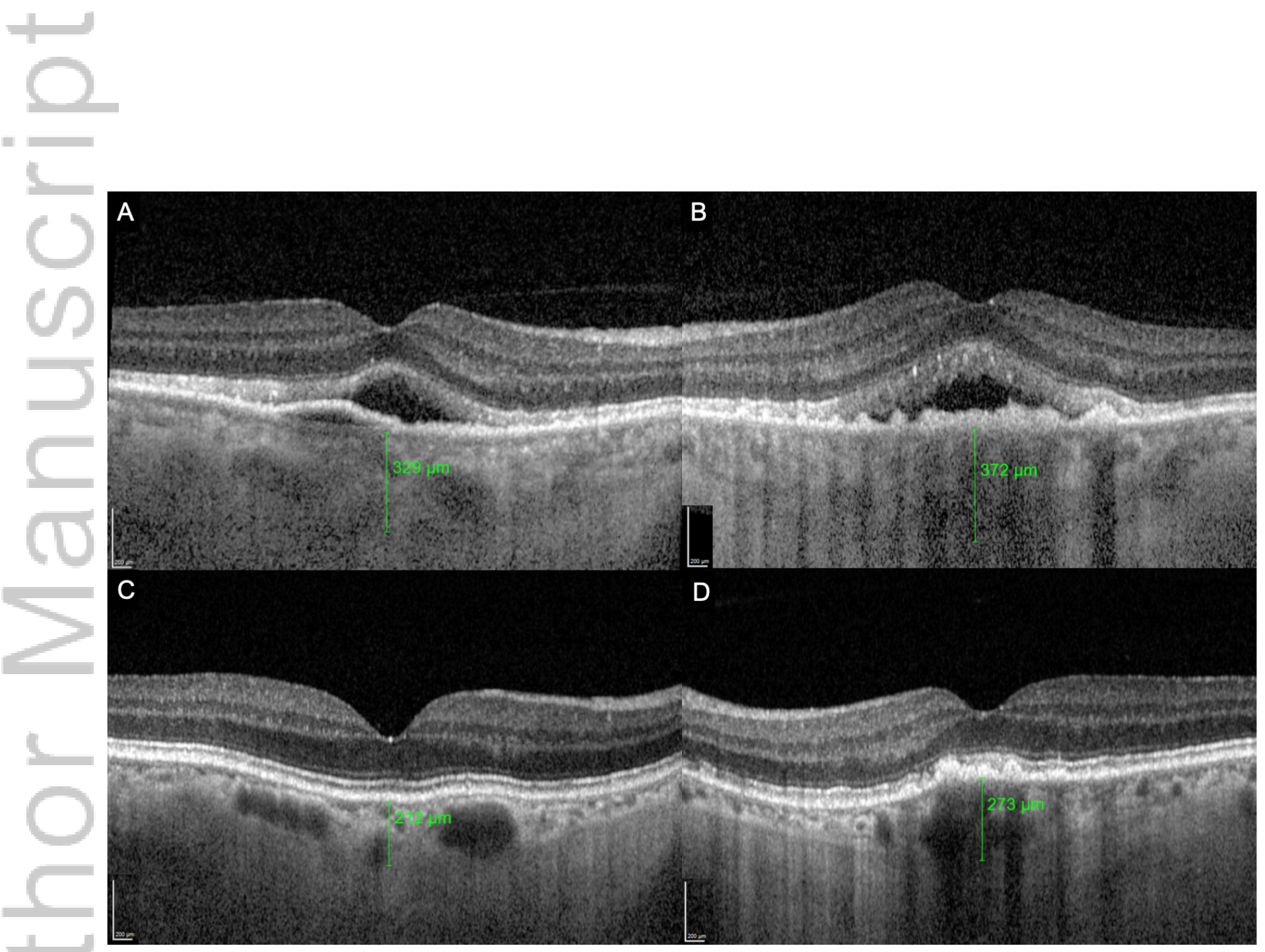

CEO_13732_CEO-19-08-0837 figure 2.tiff

This article is protected by copyright. All rights reserved. 


\section{University Library}

\section{- M M N E R VA A gateway to Melbourne's research publications}

Minerva Access is the Institutional Repository of The University of Melbourne

Author/s:

Fuzzard, DRW;Gin, TJ;Hardy, TG;Harper, CA

Title:

Subretinal fluid and choroidal venous congestion secondary to a presumed dural cavernous sinus fistula

Date:

2020-02-23

Citation:

Fuzzard, D. R. W., Gin, T. J., Hardy, T. G. \& Harper, C. A. (2020). Subretinal fluid and choroidal venous congestion secondary to a presumed dural cavernous sinus fistula. CLINICAL AND EXPERIMENTAL OPHTHALMOLOGY, 48 (4), pp.517-519. https:// doi.org/10.1111/ceo.13732.

Persistent Link:

http://hdl.handle.net/11343/275452 